\title{
Deep Sequencing of T-cell Receptor DNA as a Biomarker of Clonally Expanded TILs in Breast Cancer after Immunotherapy
}

\author{
David B. Page, 2, ${ }^{1,}$ Jianda Yuan ${ }^{1, *}$, David Redmond ${ }^{3}$, Y Hanna Wen', Jeremy C. Durack ${ }^{1}$, \\ Ryan Emerson ${ }^{4}$, Stephen Solomon', Zhiwan Dong ${ }^{1}$, Phillip Wong ${ }^{1}$, Christopher Comstock', \\ Adi Diab ${ }^{5}$, Janice Sung ${ }^{1}$, Majid Maybody ${ }^{1}$, Elizabeth Morris ${ }^{1}$, Edi Brogi ${ }^{1}$, Monica Morrow ${ }^{1}$, \\ Virgilio Sacchini ${ }^{1}$, Olivier Elemento ${ }^{3}$, Harlan Robins ${ }^{6}$, Sujata Patil ${ }^{1}$, James P. Allison ${ }^{5}$, \\ Jedd D. Wolchok ${ }^{1,3}$, Clifford Hudis ${ }^{1,3}$, Larry Norton ${ }^{1,3}$, and Heather L. McArthur ${ }^{1,3}$
}

\section{Abstract}

In early-stage breast cancer, the degree of tumor-infiltrating lymphocytes (TIL) predicts response to chemotherapy and overall survival. Combination immunotherapy with immune checkpoint antibody plus tumor cryoablation can induce lymphocytic infiltrates and improve survival in mice. We used T-cell receptor (TCR) DNA sequencing to evaluate both the effect of cryoimmunotherapy in humans and the feasibility of TCR sequencing in early-stage breast cancer. In a pilot clinical trial, 18 women with early-stage breast cancer were treated preoperatively with cryoablation, single-dose anti-CTLA-4 (ipilimumab), or cryoablation + ipilimumab. TCRs within serially collected peripheral blood and tumor tissue were sequenced. In baseline tumor tissues, T-cell density as measured by TCR sequencing correlated with TIL scores obtained by hematoxylin and eosin (H\&E) staining. However, tumors with little or no lymphocytes by H\&E contained up to $3.6 \times 10^{6} \mathrm{TCR}$ DNA sequences, highlighting the sensitivity of the ImmunoSEQ platform. In this dataset, ipilimumab increased intratumoral Tcell density over time, whereas cryoablation \pm ipilimumab diversified and remodeled the intratumoral T-cell clonal repertoire. Compared with monotherapy, cryoablation plus ipilimumab was associated with numerically greater numbers of peripheral blood and intratumoral T-cell clones expanding robustly following therapy. In conclusion, TCR sequencing correlates with H\&E lymphocyte scoring and provides additional information on clonal diversity. These findings support further study of the use of TCR sequencing as a biomarker for T-cell responses to therapy and for the study of cryoimmunotherapy in early-stage breast cancer. Cancer Immunol Res; 4(10); 835-44. @2016 AACR.

\section{Introduction}

The immune system may play a pivotal role in mediating longterm survival in women diagnosed with early-stage breast cancer. The benefits of conventional breast cancer therapies such as chemotherapy (1), human epidermal growth factor receptor 2 (HER2)-targeted therapy (2-4), and radiotherapy (5) are at least partially dependent on an adaptive immune response against the cancer. The response to chemotherapy and overall survival can be predicted by the quantity of tumor infiltrating lymphocytes (TIL)

${ }^{1}$ Memorial Sloan Kettering Cancer Center (MSKCC), New York, New York ${ }^{2}$ Providence Portland/Robert W. Franz Cancer Research Center and Earl A. Chiles Research Institute, Portland, Oregon. ${ }^{3}$ Weill Cornell Medical College, New York, New York. ${ }^{4}$ Adaptive Biotechnologies, Seattle, Washington. ${ }^{5}$ The University of Texas MD Anderson Cancer Center, Houston, Texas. ${ }^{6}$ Fred Hutchinson Cancer Center, Seattle, Washington

${ }^{*}$ D.B. Page and J. Yuan share first authorship of and contributed equally to this article.

Current address for J. Yuan: Merck Research Labs; Translational ImmunoOncology Research Merck \& Co., Inc., Rahway, NJ.

Corresponding Author: David B. Page, Providence Portland/Robert W. Franz Cancer Research Center, Earl A. Chiles Research Institute, 4805 N.E. Glisan St. North Pavilion, 2N87, Portland, OR 97213. Phone: 503-215-7807; Fax: 503-2156841; E-mail: david.page2@providence.org

doi: 10.1158/2326-6066.CIR-16-0013

C2016 American Association for Cancer Research. in tumor biopsies (6). However, not all breast cancers appear to trigger an antitumor immune response. Some breast tumors are densely infiltrated by lymphocytes, whereas most are devoid of lymphocytes by routine hematoxylin and eosin (H\&E) staining. Tumors with abundant lymphocytes grow despite immune engagement, whereas tumors with few or no lymphocytes grow in absence of immune engagement. Patients with "TIL-low" tumors, also described as "immunologically ignorant" (7) or "noninflamed" tumors (8), are more prone to chemotherapy resistance (lower pathologic complete response rates to neoadjuvant therapy) and poor overall survival $(6,9,10)$, suggesting that endogenous immune engagement may be a key survival determinant.

Immune therapies have the potential to facilitate de novo immune engagement when an endogenous immune response has not occurred. To achieve this, an optimal immune therapy would facilitate four critical components of a de novo adaptive response tumor antigen release, tumor antigen presentation, diminished immune suppression, and tumor antigen-specific T-cell activation The first components, tumor antigen release and presentation, can be facilitated by tumor cryoablation (cryo, using an image-guided probe to lyse tumor cells). The latter components, T-cell activation and T-regulatory cell depletion, can be induced by a therapeutic antibody against the T-cell coinhibitory molecule CTLA-4 $(11,12)$ In mice, the combination of these two immunotherapies, coined "cryo-immunotherapy," generated intratumoral T-cell expansion specific to tumor-associated antigens (TAA), systemic tumor regression, and improved overall survival (11) 
During cryo, the tumor is treated with repetitive freeze/thaw cycles, inducing crystallization, mechanical cellular damage, microvascular injury, ischemia, cell death, and massive protein release (13). Freezing is most efficient at the center of the probe, leading to cellular necrosis, release of inflammatory cellular contents, and activation of the innate immune system via activation of Toll-like receptor and other innate receptors. At cryoablation margins (lower isotherms), apoptotic cell death occurs, which is associated with phagocytosis and antigen presentation. Thus, cryotherapy may produce an optimal microenvironment for T-cell activation against a broad array of tumor-associated antigens.

Blocking antibodies to CTLA-4 activate T cells by binding and inhibiting CTLA-4, a T-cell surface protein that is upregulated with TCR signaling and limits T-cell activation. The CTLA-4-blocking antibody ipilimumab improves overall survival in metastatic melanoma $(14,15)$ and has been approved by the FDA for this condition. CTLA-4 blockade is thought to decrease the threshold required for T-cell activation following exposure to antigen (16), increase the quality and duration of T-cell activation, and deplete suppressive T-regulatory cells within the tumor microenvironment (17). However, immune checkpoint antibody therapy alone may not be sufficient to induce an effective antitumor immune response in all tumors. In metastatic melanomas treated with pembrolizumab, another immune checkpoint antibody against programmed death 1 (PD-1), tumors with low TILs were unlikely to respond to therapy, whereas tumors with robust TILs were more likely to respond (18). Therefore, especially in TIL-low tumors, a combination approach may increase likelihood of mounting an effective antitumor immune response.

In a post hoc analysis of a recent study (19) evaluating cryo-immunotherapy in early-stage breast cancer, we sought to determine whether cryoimmunotherapy induced broad and robust $\mathrm{T}$-cell activation and proliferation within the tumor microenvironment. Because breast cancer core biopsies are small and less conducive to comprehensive immunologic profiling by flow cytometry, and because routine histologic or immunohistochemical quantitation of $\mathrm{T}$ cells is difficult when tumor microarchitecture is disrupted by cryo, we used a novel high-throughput TCR $\beta$ (TCRB) DNA sequencing technique capable of quantifying T cells from DNA extracted from a small $(25 \mu \mathrm{m})$ tumor sample.

TCRB DNA sequencing can be used to quantify expansion of individual clones, which may serve as a surrogate marker for therapy-associated induction of antigen-specific T-cell activation. During T-cell development in the thymus, the complementaritydetermining region 3 (CDR3) region of the TCRB gene undergoes recombination and nucleotide addition/deletion steps to generate a vast pool of T-cell clones, each with a unique TCR conferring antigen specificity. If a $\mathrm{T}$ cell binds its cognate antigen and becomes activated, it undergoes clonal proliferation, allowing for an adaptive immune response against the target antigen.

In this study, TCRB DNA sequencing was performed on preand post-cryoimmunotherapy human breast cancer specimens to study the effects of cryoimmunotherapy on T cells in the tumor microenvironment. The intent was 2-fold: to evaluate TCRB sequencing as a novel biomarker in early-stage breast cancer, and to provide proof-of-concept that TCRB sequencing may be used to monitor the intratumoral and peripheral blood effects of immunotherapy in a preoperative early-stage breast cancer trial, highlighting analytical limitations as well as opportunities to integrate TCRB sequencing into immunotherapy clinical trials.

\section{Materials and Methods}

\section{Clinical trial design}

In a pilot study conducted at the Memorial Sloan Kettering Cancer Center (MSKCC) between 2012 and 2013 (MSKCC IRB\#11-202, NCT01502592), 18 women with operable earlystage breast cancer who had elected mastectomy were treated with: cryo alone (group A, $n=6$ ), single-dose ipilimumab $10 \mathrm{mg} /$ kg i.v. alone (group $\mathrm{B}, n=6$ ), or combination cryo plus ipilimumab $10 \mathrm{mg} / \mathrm{kg}$ i.v. (group C, $n=6$; Table 1 and Fig. 1). In one subject from group $\mathrm{A}$, a cryo probe malfunction occurred resulting in incomplete tumor freezing; this subject was replaced; however, the baseline core biopsy data (which were unaffected by the probe malfunction) were included in the exploratory biomarker analyses (group A total, $n=7$ ).

The treatment schedule was designed to optimize antigen exposure time without disrupting the average 2-week lead time between diagnosis and standard-of-care mastectomy. Following consent, ipilimumab (groups B and C) was administered a median of 2.5 days (range, 1-8) prior to core biopsy, cryo (groups A and C) was administered at the time of core biopsy, and mastectomy was performed a median of 7 days (range, 4-10) following core biopsy. In all subjects, core biopsy and mastectomy specimens were formalin-fixed and paraffin embedded (FFPE), and cryopreserved if additional tumor was available. Peripheral blood mononuclear cells (PBMC) collected on the day of core biopsy, and mastectomy date were cryopreserved.

\section{TCR $\beta$ CDR3 amplification and sequencing}

DNA extraction of PBMCs and FFPE-preserved biopsy/mastectomy tissue or fresh frozen tissue was performed following the DNeasy Blood and Tissue Kit (Qiagen). FFPE was used in all cases when feasible, or fresh frozen in cases where FFPE was not available (itemized in Table 1). After confirmation of the presence of tumor by pathology, a $25-\mu \mathrm{m}$ section of core biopsy or tumorbearing mastectomy block was obtained for DNA extraction. Proteinase $\mathrm{K}$ was used for digestion and extraction of DNA following a blood/cell protocol with RNase treatment and using the spin-column method. Samples were analyzed by highthroughput sequencing of the TCR $\beta$ CDR3 region using the ImmunoSEQ immune profiling system at the deep level (Adaptive Biotechnologies; ref. 20). Sequencing was performed on an Illumina HiSeq system (Illumina). This analysis results in a mean of approximately $5 \times$ sequence coverage for T cells from $3.6 \mu \mathrm{g}$ of genomic DNA; in a typical blood sample this equates to $\sim 1,000,000$ sequencing reads from $\sim 200,000 \mathrm{~T}$ cells, depending on the proportion of $\mathrm{T}$ cells. The product was sequenced and organized, providing productive and nonproductive sequences (CDR3 regions explicitly encoding a premature stop, and those predicted to put the receptor gene out-of-frame downstream of the CDR3 rearrangement, were considered nonproductive). An algorithm was applied to raw sequencing data for collapsing PCR and sequencing errors, resulting in unique rearrangements of the CDR3 genes. Productive TCR $\beta$ CDR3 sequences are the object of this study.

Quantification of TILs by staining and immunohistochemistry (IHC)

In addition to TCR sequencing, adjacent tissue from FFPE tumor core biopsies and resection specimens were sectioned with a standard thickness of $5 \mu \mathrm{m}$ and visualized by routine $\mathrm{H} \& \mathrm{E}$ 
Table 1. Tumor characteristics and TCR deep-sequencing metrics, by patient

\begin{tabular}{|c|c|c|c|c|c|c|c|c|c|c|c|}
\hline \multirow[b]{2}{*}{ Group } & \multirow[b]{2}{*}{ ID } & \multirow[b]{2}{*}{ Subtype } & \multirow[b]{2}{*}{ Diff } & \multicolumn{2}{|c|}{ Days duration between } & \multirow{2}{*}{$\begin{array}{c}\text { H\&E } \\
\text { TIL Score } \\
(\%)\end{array}$} & \multicolumn{5}{|c|}{ Bx T-cell Receptor Deep Sequencing Metrics } \\
\hline & & & & $\mathbf{p} \mathbf{p} \rightarrow \mathbf{B x}$ & $\mathrm{Bx} \rightarrow$ Surgery & & $\begin{array}{l}\text { Sample } \\
\text { Source }\end{array}$ & $\begin{array}{l}\text { Absolute \# } \\
\text { Sequences }\end{array}$ & $\begin{array}{l}\text { \# Unique } \\
\text { Sequences }\end{array}$ & Density & Clonality \\
\hline \multirow[t]{7}{*}{ Cryoablation } & 1 & $\mathrm{HR}^{+}$ & mod & & 10 & $<5$ & FFPE & 2193838 & 16895 & 30 & 0.11 \\
\hline & 2 & $\mathrm{HR}^{+}$ & $\bmod$ & & 8 & $<5$ & Frozen & 1209573 & 7909 & 7 & 0.20 \\
\hline & 3 & $\mathrm{HR}^{+}$ & poor & & 8 & $<5$ & Frozen & 1453469 & 14020 & 5 & 0.12 \\
\hline & 4 & $\mathrm{HR}^{+}$ & $\bmod$ & & 8 & 85 & FFPE & 867803 & 49283 & 24 & 0.23 \\
\hline & $5^{\mathrm{a}}$ & $\mathrm{HR}^{+}$ & $\bmod$ & & 9 & $<5$ & FFPE & 652336 & 2974 & 5 & 0.13 \\
\hline & 6 & $\mathrm{HR}^{+}$ & well & & 7 & n/a & Frozen & 55054 & 647 & 2 & 0.12 \\
\hline & 7 & $\mathrm{HR}^{+}$ & well & & 7 & $<1$ & FFPE & 82343 & 3467 & 5 & 0.12 \\
\hline \multirow[t]{6}{*}{ Ipilimumab } & 8 & $\mathrm{HER}^{+}$ & poor & 8 & 4 & 80 & FFPE & 5278898 & 67770 & 19 & 0.14 \\
\hline & 9 & TNBC & poor & 1 & 7 & $<5$ & FFPE & 205971 & 755 & 3 & 0.13 \\
\hline & 10 & $\mathrm{HR}^{+}$ & well & 3 & 7 & $<5$ & Frozen & 694364 & 2651 & 0 & 0.41 \\
\hline & 11 & $\mathrm{HR}^{+}$ & poor & 5 & 8 & n/a & FFPE & 164476 & 697 & 2 & 0.17 \\
\hline & 12 & HER2 $^{+}$ & poor & 1 & 7 & 40 & FFPE & 8916191 & 33101 & 16 & 0.23 \\
\hline & 13 & TNBC & poor & 1 & 8 & 10 & FFPE & 368214 & 13966 & 15 & 0.11 \\
\hline \multirow[t]{6}{*}{ Cryoablation + Ipilimumab } & 14 & $\mathrm{HR}^{+}$ & $\bmod$ & 3 & 8 & $<5$ & FFPE & 2714728 & 11751 & 6 & 0.15 \\
\hline & 15 & TNBC & poor & 1 & 7 & 40 & FFPE & 3479585 & 14414 & 10 & 0.16 \\
\hline & 16 & $\mathrm{HR}^{+}$ & poor & 2 & 6 & 5 & FFPE & 235779 & 9486 & 13 & 0.13 \\
\hline & 17 & $\mathrm{HR}^{+}$ & poor & 4 & 7 & 30 & FFPE & 429163 & 12035 & 7 & 0.14 \\
\hline & 18 & $\mathrm{HR}^{+}$ & $\bmod$ & 2 & 10 & $<5$ & FFPE & 1397054 & 5412 & 5 & 0.12 \\
\hline & 19 & $\mathrm{HR}^{+}$ & mod & 5 & 7 & $<5$ & Frozen & 583965 & 7382 & 4 & 0.10 \\
\hline Median & & & & 2.5 & 7 & $<5$ & & 781084 & 10619 & 6 & 0.14 \\
\hline
\end{tabular}

NOTE: The reported metrics describe the research core biopsy, obtained at the time of cryoablation (arms A and C) and shortly after ipilimumab (arms B and C). Due to limited specimen availability, experiments were only repeated when sequencing did not meet quality control standards (refer to Materials and Methods). Abbreviations: Diff, differentiation; Ipi, ipilimumab; Bx- biopsy; n/a- not available for analysis.

an patient 5, cryoablation probe malfunction precluded interpretation of therapy effect; however, the core biopsy was still evaluable.

staining. Quantity of stromal TILs was measured by a trained pathologist using the San Antonio 2014 TILs Working Group 2014 guidelines (6).

\section{Analysis of duplicates}

Because of the post hoc nature of this experiment, sufficient material was not available from all specimens to allow for duplicate analysis. In select specimens where the total DNA content was low and additional tissue was available, the assay was repeated. The larger (greater absolute TCRB sequence count) specimen was used for analysis, except in cases where the absolute sequence count differed by less than a factor of 10 , in which case the duplicates were averaged.

\section{Statistical analysis}

Analyses were performed using Microsoft Excel, ImmunoSEQ software (Adaptive Biotechnologies), GraphPad prism (GraphPad Software), and the Rstatistical software. Because of the post hoc nature of this study, most analyses are descriptive for the purpose of generating hypotheses. A limited number of findings were evaluated for statistical significance: groups were compared using a nonparametric two-sided Mann-Whitney test, and correlations were evaluated assuming a non-Gaussian distribution (Spearman correlation), unless otherwise indicated.

\section{Results}

TCRB sequencing measures T-cell infiltration in early-stage breast cancer biopsies

In previous series of early-stage breast cancer patients, TIL counts as measured by routine H\&E staining are nonnormally distributed, with most tumors exhibiting sparse or no TILs (as measured by $\leq 10 \%$ of stroma, $69 \%$ of triple-negative tumors in a recent report), and the minority of tumors exhibiting dense TILs (comprising upward of $80 \%$ of the stromal tissue examined; ref. 21). In our dataset using the standardized H\&E TIL scoring methodology proposed by the San Antonio TIL Working group, we found the same nonnormal distribution (Table 1), with the majority of tumors having TIL count $\leq 10 \%$ (6).

As an alternative to $\mathrm{H} \& \mathrm{E}$, we characterized the degree of T-cell infiltrates in core biopsies using two TCRB sequencing methodologies. The first methodology, absolute T-cell count, is a direct measure of the number of detectable T-cell DNA sequences within the specimen. The second methodology, T-cell density, is a relative measure of T-cell DNA relative to total DNA (including tumor and stromal DNA), which may be more stable to variations in sequencing depth. In our series, both absolute T-cell count and T-cell density exhibited a nonnormal distribution similar to H\&E TIL count. Absolute T-cell sequence counts ranged from $5.5 \times 10^{4}$ to $8.9 \times 10^{6}$ sequences per sample (mean $1.98 \times 10^{6}$; $\mathrm{SD}, 2.68 \times 10^{6}$ ), and T-cell densities ranged from 0 to $30 \%$ (mean
Figure 1.

Study design.

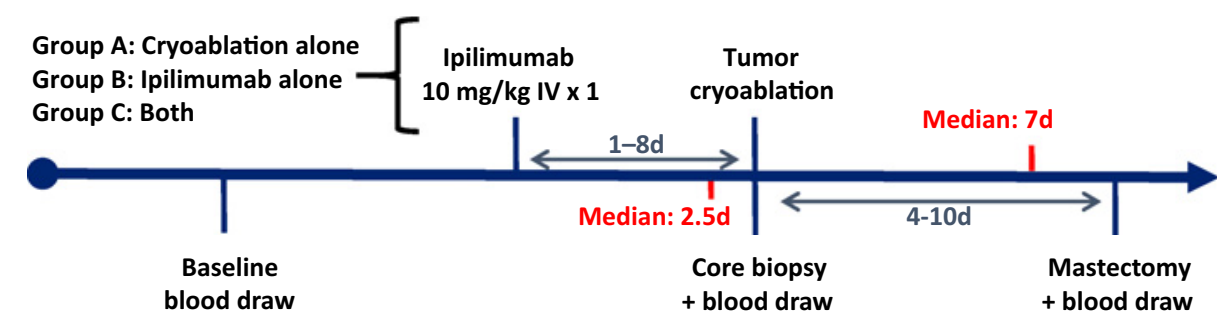



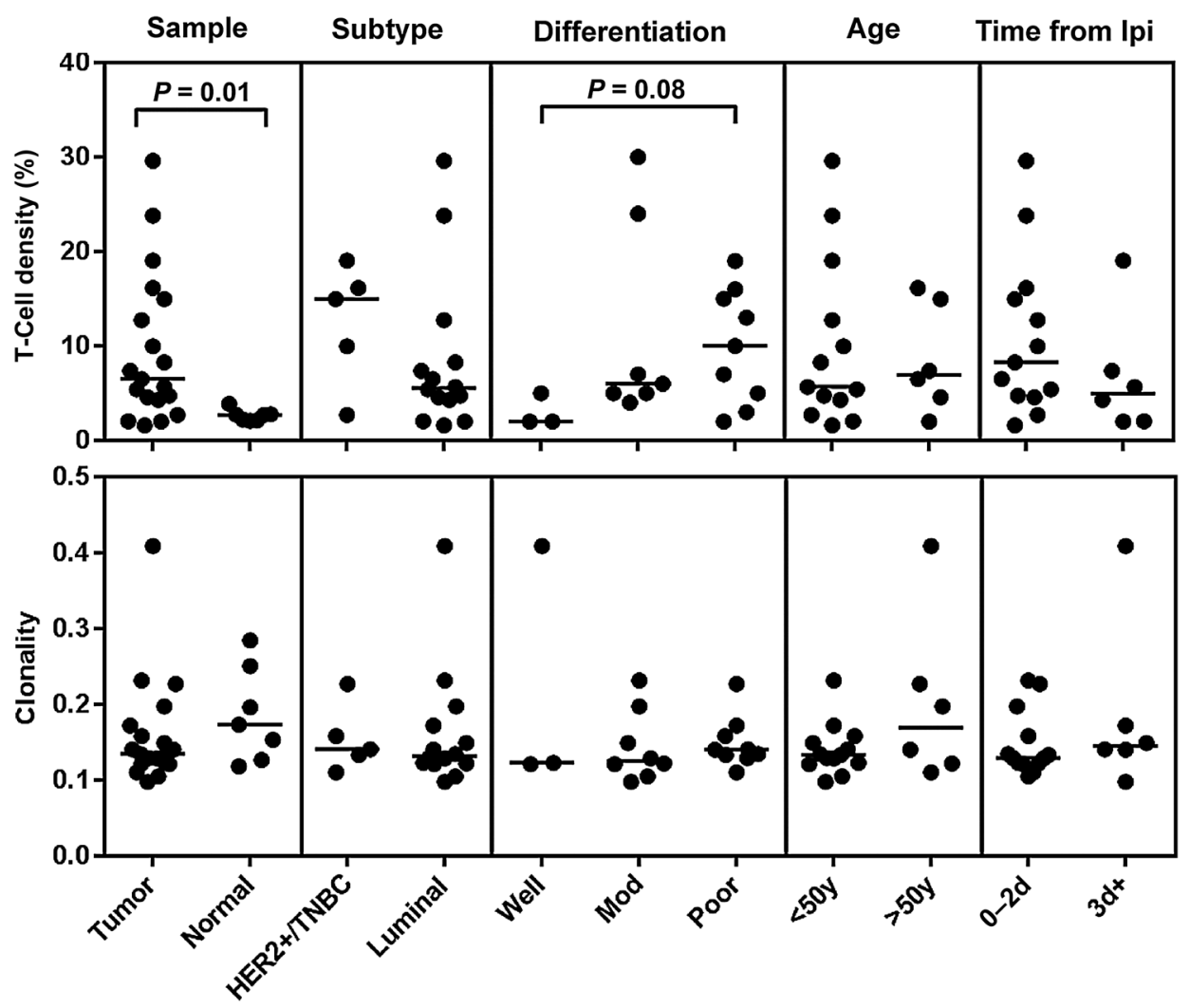

Figure 2.

Influence of patient and tumor characteristics on core biopsy T-cell infiltrates. T-cell density and clonality are assessed by TCR DNA sequencing with group medians depicted as solid lines. Unless specified, differences across groups are not statistically significant. T-cell density in untreated normal breast tissue was lower compared with tumor; HER2 ${ }^{+}$and TNBC subtypes appear to have denser infiltrates compared with luminal-type (i.e., $\mathrm{HR}^{+} / \mathrm{HER2} 2^{-}$); tumor grade is associated with T-cell density; age is not associated with T-cell density or clonality; and subjects receiving ipilimumab greater than 3 days preceding biopsy did not have appreciable differences in T-cell density or clonality compared with subjects not receiving ipilimumab, or receiving ipilimumab within $0-2$ days. HER2 ${ }^{+}$, human epidermal growth factor receptor 2 positive; TNBC, triple-negative breast cancer; mod, moderate; Ipi, ipilimumab.
5.7\%; SD 8.1\%). T-cell density was significantly correlated with TIL count by H\&E $(r=0.64, P=0.007)$. There was a trend toward correlation between absolute T-cell count and H\&E $(r=0.46, P=0.06)$.

We conducted TCRB sequencing of normal adjacent breast tissue specimens (at least $3 \mathrm{~cm}$ away from tumor) obtained during mastectomy. In subjects who did not receive ipilimumab (i.e., patients undergoing cryo alone, $n=7$ ), the T-cell densities and absolute T-cell counts of normal breast tissue were lower and more uniformly distributed compared with tumor (mean/SD T-cell density: tumor, 5.7\%/8.1\%; normal, $2.3 \% / 1.1 \%, P=0.002)$. In subjects who received ipilimumab (i.e., ipilimumab alone and ipilimumab/cryo arms), normal breast tissue appeared to have more dense and less uniformly distributed T-cell infiltrates, similar to the tumoral specimens (mean/SD T-cell density: tumor 7.9\%/8.5\%). Samples with few or no apparent TILs by H\&E $(\leq 5 \%)$ exhibited a median of 301,603 T-cell sequence counts by TCRB sequencing (range, 42,126 to $3.6 \times 10^{6}$ ).

The small size of this study precluded definitive assessment of the influence of tumor/patient characteristics on core biopsy T-cell infiltrate quantity. However, Fig. 2 illustrates the potential influence of various factors on baseline T-cell infiltrate quantity. Both tumor differentiation and tumor subtype appeared to influence Tcell infiltrate quantity, with higher grade lesions and TNBC or HER2 $^{+}$lesions having denser infiltrates. Ipilimumab was administered prior to the core biopsy (median 2.5 days prior), and hypothetically this may have influenced T-cell infiltrates in this biopsy. However, subjects who received ipilimumab more than 3 days prior to biopsy $(n=6)$ did not demonstrate appreciable differences in infiltrate quantity compared with subjects not receiving ipilimumab or receiving ipilimumab within 2 days of biopsy (Fig. 2).

\section{Induction of T-cell polyclonality and clonal repertoire} remodeling

TCR sequencing can also assess the clonal nature of infiltrating $\mathrm{T}$ cells, i.e., whether the infiltrates are comprised of a few highfrequency clones (oligoclonal) versus many unique low-frequency clones (polyclonal). In our dataset, the number of unique T-cell clonal populations within a sample correlated with the absolute T-cell count ( $\mathrm{r}=0.76, P=0.0002)$. To assess clonal diversity independent of this observed correlation, we calculated the Shannon entropy of the specimen and normalized it to the number of unique clones within the sample. This previously described "clonality" metric ranges from 0 to 1 and is relatively insensitive to sample size, with 1 representing a purely monoclonal population, versus 0 representing a purely polyclonal population (22). Using this metric, clonality of the core biopsy specimens ranged from 0.10 to 0.41 (mean 0.16 ; SD 0.07), which was similar in degree and variation to normal breast tissue T-cell clonality (mean 0.18; SD 0.07). In this small dataset, T-cell clonality did not appear to be associated with subject age, tumor pathologic grade, or HR/HER2 subtype (Fig. 2).

To determine the intratumoral effects of cryo and/or singledose ipilimumab, we conducted T-cell repertoire analysis on matched core biopsies and mastectomies. Effects on absolute count varied widely within treatment groups (data not shown). Cryo decreased T-cell density in the majority (5/6) of subjects conversely ipilimumab alone increased T-cell density in the majority $(5 / 6)$ of subjects, whereas no trend was observed following combination therapy (Fig. 3). We then evaluated the effect 

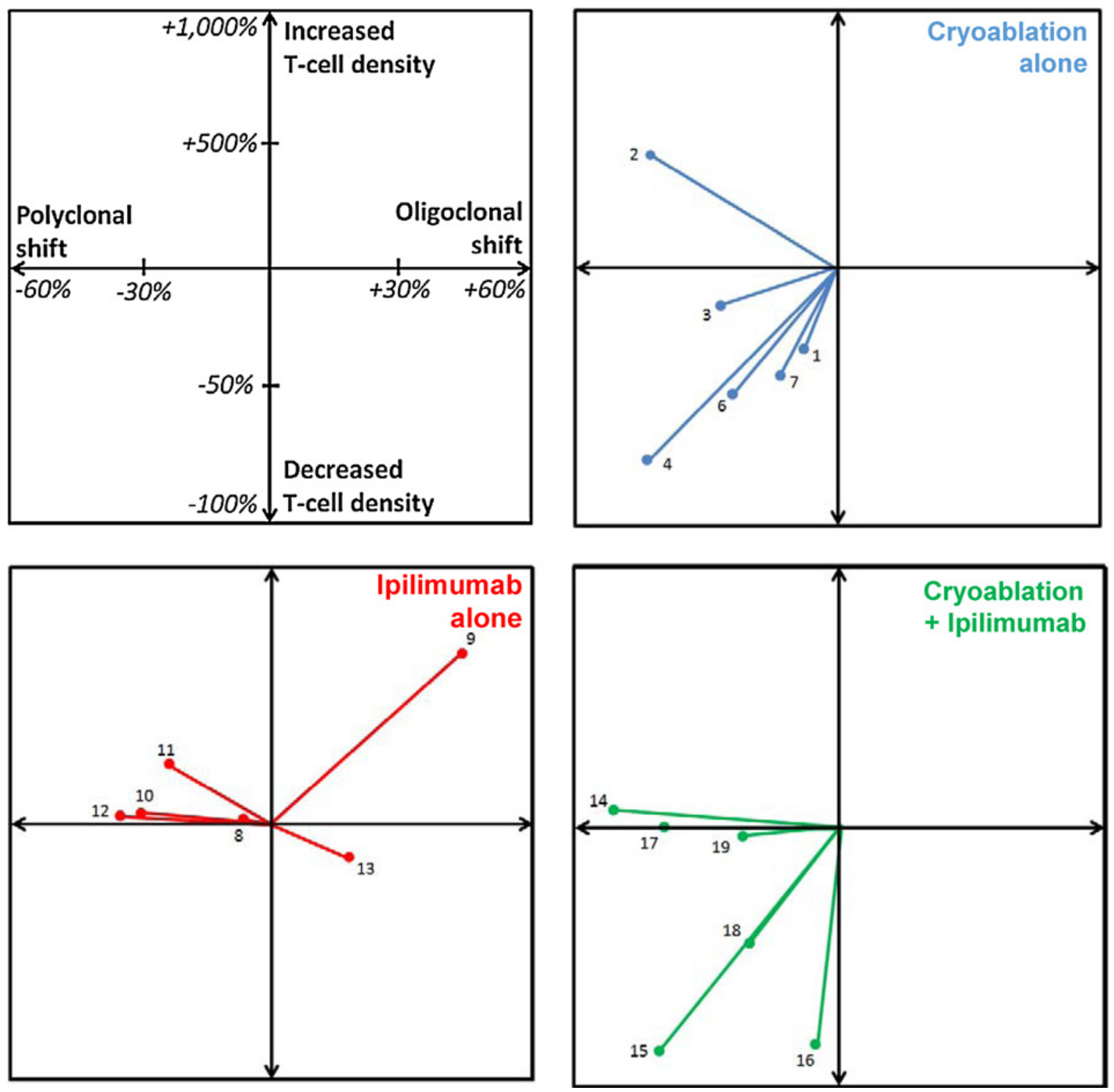

Figure 3.

Effect of therapy on intratumoral T-cell density and clonality by TCR deep sequencing. T-cell density and clonality are depicted as fold change, comparing core biopsy to mastectomy. The $x$-axis depicts fold change in T-cell clonality, with positive values indicating a shift toward oligoclonal T-cell infiltrates; the $y$-axis depicts fold change in T-cell density, with positive values indicating a shift toward denser T-cell infiltrates. Each line/dot demonstrates an individual patient treated on protocol, and numbers indicate patient identifier (as listed in Table 1).

of therapy on clonality. One hundred percent of cryoablated tumors $(n=12)$, irrespective of ipilimumab, experienced a shift toward polyclonality (median clonality change: $-0.04 ; P=$ 0.005), whereas no trend in clonality was observed following ipilimumab alone. These findings are graphically depicted in Fig. 3, which illustrates the change in T-cell clonality ( $x$-axis) and the change in T-cell density ( $y$-axis) for each patient, according to treatment arm (Fig. 3).

Next, we evaluated whether ipilimumab and/or cryo remodels the clonal repertoire, i.e., whether new clones are introduced or the distribution of clones is modified. Morisita's overlap is a previously described (23) metric that measures the proportion of shared T-cell sequencing reads over two samples, normalizing for biases introduced by sample size. This overlap metric ranges from 0 to 1 , with low overlap scores indicating repertoire remodeling and potential influx of novel T-cell clones. Relative to the ipilimumab-alone group, we found a trend of lower Morisita's overlap in cryo-treated groups $(P=0.08)$, suggesting greater remodeling of the intratumoral clonal repertoire following cryo. In summary, these findings suggest that ipilimumab alone expands intratumoral lymphocytes, cryo alone contracts intratumoral lymphocytes and induces polyclonality and repertoire 

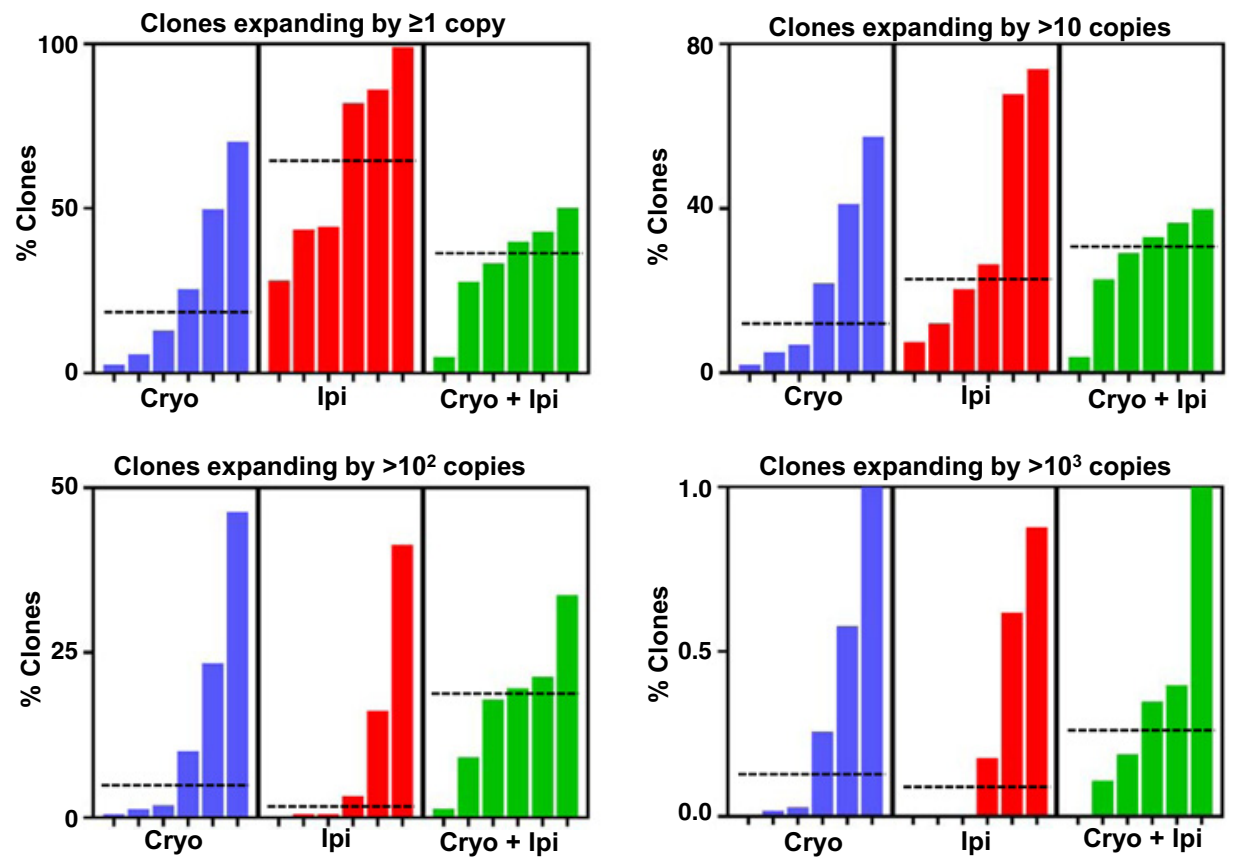

Figure 4.

Effect of therapy on clonal expansion. Each graph depicts the proportion of intratumoral T-cell clones expanding by the specified threshold (i.e., expansion by $>1$ copy, $>10$ copies, $>100$ copies, or $>1,000$ copies), comparing core biopsy to mastectomy. Each solid line represents an individual patient, with dotted lines representing group medians. Legend: Cryo-cryoablation; Ipi, ipilimumab. remodeling, and the combination induces polyclonality and repertoire remodeling with less lymphocytic contraction.

Induction of high-magnitude intratumoral T-cell clonal expansions

Certain T-cell clones, when exposed to released antigens following cryo, might expand more robustly with the addition of ipilimumab. Therefore, we calculated the pre-/post-treatment change in abundance of each individual T-cell clone found in either the core biopsy or mastectomy specimen. We then quantified the number of clones that expanded with therapy by certain absolute sequence count thresholds, and normalized this number by the total clone count. Using this technique with the threshold set to $\geq 1$ copy (thus measuring the proportion of clones expanding by any magnitude), we found that the greatest percentage of T-cell clones expanded with ipilimumab alone (median \% clones expanding: $18 \%$ or 4,454 clones, cryo; $62 \%$ or 41,399 clones, ipilimumab, $36 \%$ or 4,307 clones, cryo + ipilimumab). However, if we repeated this with the threshold set to 10 , we found that the greatest percentage of T cells expanded with cryo plus ipilimumab (median \% clones expanding: $14 \%$ or 2,822 clones, cryo; $23 \%$ or 11,322 clones, ipilimumab, $31 \%$ or 3,658 clones, cryo + ipilimumab). At higher thresholds $\left(10^{2}\right.$ or $\left.10^{3}\right)$, the differences appear more pronounced (Fig. 4), indicating that more high-magnitude T-cell expansions were observed in the tumors treated with combination ipilimumab plus cryo, relative to monotherapy.

Peripheral blood clonal expansions are less discernible than TIL clonal expansions

In baseline tumor and matched PBMC samples acquired at time of biopsy, we found a 5 -fold greater number of unique clonal Tcell populations (range, -1- to 232-fold) in matched peripheral blood specimens compared with TILs. Normalizing the clonal diversity of PBMCs for sample size using the clonality metric, PBMCs were only modestly more polyclonal than T cells obtained from biopsy (13/18 subjects, 0.02 median difference in clonality).
We then evaluated the effect of therapy on peripheral repertoire by analyzing serially collected PBMCs. In subjects receiving cryo alone $(n=6)$, two pretreatment time points were analyzed (at screening and within 1 day preceding cryo), allowing for characterization of baseline intrapatient variations in repertoire over time in the absence of intervening therapy. The clonality metric exhibited a low degree of intrapatient variation (median difference between samples 0.02), whereas measures of T-cell quantity (absolute count and T-cell density) were prone to wide intrapatient variation. We then evaluated the effect of therapy over time, comparing baseline to posttreatment peripheral blood obtained during the 30-day safety visit. We found no obvious trends with either of the three arms over time in T-cell clonality, absolute T-cell quantity, or T-cell density (data not shown). We then evaluated the Morisita's overlap of pretreatment versus posttreatment peripheral blood and found no appreciable differences in overlap across the three treatment groups.

Using the same method described for TILs, we then calculated the pretreatment/posttreatment change in abundance of each individual T-cell clone found in the peripheral blood specimens. Peripheral blood concentrations of T-cell clones are likely to fluctuate to some degree over time, in the absence of therapy. To assess the degree of fluctuation of clonotype concentration, we compared the two pretreatment time points of the cryo-treated subjects. In the absence of therapy, roughly half of clones expanded (median $45 \%$ ) from time point A (screening) to time point $\mathrm{B}$ (preceding ablation). However, only $\sim 1 \%$ of clones expanded by an absolute count of $10 \%$ to $100, \sim 1 \%$ of clones expanded by counts of 100 to 1,000 , and virtually no clones expanded by $>1,000$ counts. We then characterized the effect of ipilimumab and/or cryo on peripheral T-cell clone expansion by comparing time points B and C for each of the treatment arms. The degree of clonal expansion was similar to the untreated time points, with the exception of the combined ipilimumab + cryo arm, which exhibited a greater proportion of clones expanding by $>100$ (median \% 
Figure 5.

Relationship of intratumoral versus peripheral blood T-cell clonal expansion. Each graph represents the $\mathrm{T}$-clonal repertoire of a representative patient of each of the three treatment groups. Each point represents a unique T-cell clone detectable in the core biopsy, mastectomy, and/or peripheral blood. The $x$-axis depicts the absolute change in intratumoral clonal frequency count, comparing core biopsy to mastectomy. The $y$-axis depicts the absolute change in peripheral blood clonal frequency count, comparing pretreatment) to post-treatment time points. For example, points in the upper right quadrant depict T-cell clones that have increased in frequency both within the tumor and in the peripheral blood.
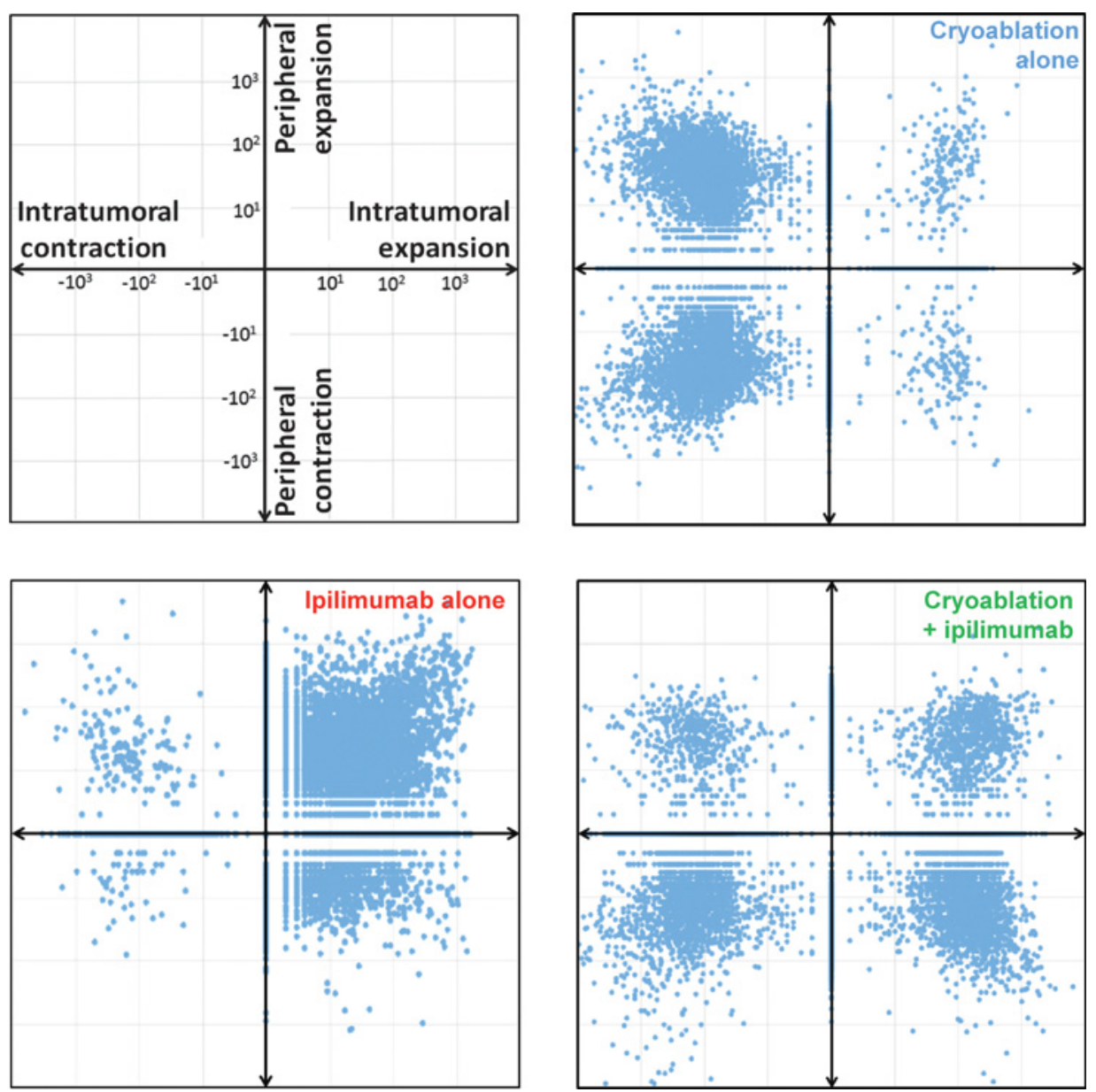

of expanding clones: untreated, $1 \%$; cryo, $1 \%$, ipilimumab, $1 \%$; cryo/ipilimumab, 6\%). These high-magnitude clonal expansions corresponded to a median absolute number of 1,253 clones per treated patient in the ipilimumab + cryo arm.

\section{Independent intratumoral and peripheral TCR repertoire} landscapes

Assuming that TILs are exposed to tumor-associated antigens and therefore enriched for tumor-reactive T-cell clones, the more contracted intratumoral clonal repertoire could be used to identify and monitor tumor-reactive clones within the peripheral blood. We tested the hypothesis that intratumoral T-cell expansions might be mirrored in peripheral blood by categorizing intratumoral clonotypes into groups by degree of intratumoral expansion, using the previously defined thresholds ( $>10$ copies, $>100$ copies, and $>10^{3}$ copies). We then evaluated the degree of expansion of each of these clonotype groups within the peripheral blood over time, comparing baseline to 30-day posttreatment follow-up. Most of the clones expanding within the tumor were undetectable in the peripheral blood, either before or after therapy. Regardless of the degree of intratumoral clonal expansion, only a low percentage of clonotypes were also expanding in the peripheral blood, and was not influenced by the degree of intratumoral expansion (median proportion of peripherally expanding clones across all groups: $>10$ copies, $6 \%$; $>100$ copies, $7 \%)$.
We conducted graphical analyses of intratumoral versus peripheral blood clonotype expansion, plotting the absolute intratumoral expansion/contraction on the $x$-axis, versus peripheral expansion/contraction on the $y$-axis (Fig. 5). Using this technique, we found no correlation between intratumoral clonal expansion and peripheral blood clonal expansion; however, we observed several general trends across the three treatment groups. First, cryo alone appeared to induce intratumoral contraction of the majority of T-cell clones, with very little effect on peripheral blood clones (as represented by the asymmetry across the $y$-axis and symmetry across the $x$-axis). Next, ipilimumab alone appeared to induce expansion of clonotypes both in the peripheral blood and in the tumor (as represented by a dominance of clones in the double-positive quadrant). Finally, combination ipilimumab/cryo induced a mixed contraction and expansion of intratumoral clones, as represented by symmetry across the $\gamma$-axis. Furthermore, the median degree of expansion with ipilimumab/ cryo was shifted rightward, indicating that a greater proportion of clones experienced high-magnitude expansions, compared with ipilimumab alone.

\section{Conclusion}

The two goals of this post hoc analysis were to explore the potential utility of TCRB sequencing as an immune-based biomarker in early-stage breast cancer and to characterize the effect of 
cryo-immunotherapy on intratumoral and peripheral blood T-cell proliferation. We first compared TCRB sequencing to the current gold-standard immune biomarker in early-stage breast cancer, TIL count by H\&E staining. Recently, a standardized method of assessing TILs by H\&E has been developed and was validated to be predictive and prognostic in triple-negative breast cancer (6). Despite its utility and simplicity, the H\&E TIL score provides only a semiquantitative assessment on immune infiltration and poorly discerns the highest-risk patients (with the highest risk low-TIL group exhibiting relapse rates of only $30 \%$ in a recent series; ref. 21). TCRB sequencing is an attractive alternative because it quantifies $\mathrm{T}$ cells (the immune cell type most attributable protective immunity) and characterizes T-cell clonal diversity, which might be useful to further stratify risk. In our dataset, we found that the T-cell density, a method of quantifying T cells by TCRB sequencing, correlates with the gold-standard TIL assessment by H\&E. Furthermore, the distribution of TIL quantity (with most tumors having low T-cell density), and the association of T-cell density with poorly differentiated and HER2 ${ }^{+} / \mathrm{TNBC}$ tumors, closely resembles the H\&E TIL quantity metric $(21,24)$. One primary difference, however, is that TCR sequencing could quantify up to millions of T-cell DNA sequences even in samples with no TILs or low TILs by H\&E. Some of these sequences may arise from non-tumor-bearing breast tissue or vasculature within the sequenced sample; nevertheless, these data suggest that TCR sequencing could be a potentially more sensitive method of measuring intratumoral T-cells. Although not addressed by this dataset, it is conceivable that a more precise quantification of $\mathrm{T}$ cells could be helpful in stratifying the highest-risk tumors amongst tumors with low TILs by H\&E. The correlation between H\&E TIL score and T-cell density was imperfect: discordant scores may be related to intratumoral heterogeneity, or the preponderance of non-T-cell lymphocytes in some specimens, which would result in a higher H\&E TIL score but a lower T-cell density.

Our second goal was to demonstrate how TCRB sequencing could be used to characterize the effects of immunotherapy in serially collected intratumoral and PBMC specimens. We used two TCRB sequencing metrics, T-cell density and T-cell clonality, to characterize the effects of cryo and/or ipilimumab over time in tumors. T-cell clonality describes whether T-cell populations are oligo-clonal (i.e., reacting to the one or a few antigens) or polyclonal (reacting to many different antigens). In one trial, melanomas with oligoclonal T-cell infiltrates were more likely to respond to immunotherapy with anti-PD-1 (pembrolizumab; ref. 18), potentially because the dominant T-cell clones were tumor specific and amenable to reinvigoration with anti-PD-1. In our dataset, cryo alone appeared to deplete T cells but induced a polyclonal shift (Fig. 3). The observed decrease in T-cell density is consistent with previous observations that cryo induces thermal necrosis and apoptosis (of both tumors and TILs; ref. 25). The polyclonal shift and the low overlap between pretreatment and posttreatment clones suggest that new T-cell clones are infiltrating the tumor bed and are reactive to a broader variety of antigens compared with the pretreatment resident $\mathrm{T}$ cells. The polyclonal shift, however, argues against the presence of dominant, highmagnitude clonal expansions associated with a robust response against the tumor.

In the ipilimumab \pm cryo arms, ipilimumab was administered a median of 2.5 days before the research biopsy \pm cryo, and surgical mastectomy was performed a median of 7 days thereafter. This provided a unique opportunity to assess serial changes following ipilimumab \pm cryo, with the limitation that early post-ipilimumab changes (i.e., during the short window between ipilimumab and biopsy) would not be captured in this analysis. Ipilimumab appeared to expand tumor-infiltrating T cells, which is consistent with previously described observations that immunologic checkpoint blockade could induce intratumoral T-cell expansion (7). Cryo + ipilimumab had a mixed effect on T-cell density, suggesting that neither ipilimumab nor cryo were dominant in their effects on T-cell density.

We hypothesized that cryo + ipilimumab may generate more T-cell expansions relative to cryo alone; however, as illustrated in Fig. 3, cryo + ipilimumab produced a polyclonal shift similar to cryo alone. Therefore, we more closely evaluated intratumoral clonal T-cell expansion by comparing the concentration of individual T-cell clones before and after therapy. By this approach, we observed that cryo + ipilimumab was associated with a greater number of high-magnitude clonal expansions relative to monotherapy. If confirmed, this finding would suggest that cryo + ipilimumab mediates rapid proliferation of a small subset of clones. This analytic method of clonotype expansion was more useful in this dataset than the clonality metric, for discerning differences across treatment groups. Although clonal TIL expansion does not necessarily indicate an antitumor response, one would expect clonal TIL expansion in the setting of T-cell responses against tumor-associated antigens. Therefore, we argue that this metric may be a candidate biomarker for assessing therapy-associated clonal proliferation in preoperative immunotherapy clinical trials.

We also evaluated the utility of serial TCRB sequencing of PBMCs. In our paired analysis of blood and tumor, we found an increase in the number of peripherally expanding clones associated with combination therapy, but found little evidence in support of an association of intratumoral clonal change with peripheral blood clonal change. One explanation is that intratumorally expanding T-cell clones are diluted in PBMCs by the more diverse and abundant peripheral blood clonal repertoire, and may be difficult to track serially over time in PBMCs in early-stage disease. However, it is conceivable that in metastatic disease, intratumorally expanding T-cell clones expand peripherally after exposure to additional tumor antigen, and therefore may be easier to detect in the blood or in satellite metastases.

Because the analysis was post hoc with no explicit statistical analyses or adjustments for multiplicity, because the histologies of the treated tumors varied and were imbalanced across groups, and because the timing of ipilimumab was heterogeneous, we are limited in our ability to generalize our characterization of breast cancer TILs. Also, our observations regarding the clonal effects of ipilimumab \pm cryo must be confirmed prospectively in a study with more standardization of variables such as biopsy timing and specimen processing. In such a trial, more information could be derived by characterizing T-cell repertoire changes across various subsets of T cells (for example, in effector T cells, helper $\mathrm{T}$ cells, and regulatory $\mathrm{T}$ cells, which could be sorted by flow cytometry), or within draining lymph nodes, which might provide additional information regarding priming and expansion of $\mathrm{T}$ cells following cryo-immunotherapy.

In metastatic clinical trials, immune checkpoint blockade with anti-PD-1/anti-PD-L1 is modestly effective across all metastatic breast cancer subtypes (26-29). Therefore, we anticipate heightened investigation of novel combination strategies as a means of improving efficacy. We also anticipate increasing utilization of 
preoperative clinical trials, on the basis that serial analysis of breast cancer tissue may inform drug development and understanding of underlying mechanism. In conclusion, we have demonstrated that TCRB sequencing is feasible to conduct in earlystage breast cancer using small core biopsy specimens, and that the TCRB T-cell density metric correlates with the current goldstandard immune biomarker, TIL count by H\&E. We also illustrated how multi-dimensional data obtained from TCRB sequencing could elucidate the effects of immunotherapy on TILs and PBMCs, and may be informative as a biomarker in preoperative clinical trials.

\section{Disclosure of Potential Conflicts of Interest}

D.B. Page reports receiving a commercial research grant from Merck, is a consultant/advisory board member for Peregrine Pharmaceuticals and Celgene, and has received expert testimony from an entity. J. Yuan is Director, Translational Immuno-Oncology Research at Merck \& Co Inc. and has ownership interest in the TCR patent with Adaptive Technologies. S.B. Solomon reports receiving a commercial research grant from GE Healthcare and is a consultant/ advisory board member for Medtronic. P. Wong is a consultant/advisory board member for Merck. H. Robins is CSO at Adaptive Biotechnologies and has ownership interest (including patents) in the same. J.P. Allison has ownership interest (including patents) in Jounce Therapeutics, Neon, Merck, and Jounce and is a consultant/advisory board member for Kite, Amgen, Jounce, and Neon. J.D. Wolchok reports receiving commercial research grant from Bristol-Myers Squibb, Merck, Medimmune, and Genentech and is a consultant/advisory board member for Bristol-Myers Squibb, Merck, Medimmune, and Genentech. H.L. McArthur reports receiving commercial research grant from Merck, Eli Lilly, and MedImmune/Astra Zenica, other commercial research support from BristolMyers Squibb, and is a consultant/advisory board member for Merck. No potential conflicts of interest were disclosed by the other authors.

\section{References}

1. Hannani D, Locher C, Yamazaki T, Colin-Minard V, Vetizou M, Aymeric L, et al. Contribution of humoral immune responses to the antitumor effects mediated by anthracyclines. Cell Death Differ 2014;21:50-8.

2. Park S, Jiang Z, Mortenson ED, Deng L, Radkevich-Brown O, Yang X, et al The therapeutic effect of anti-HER2/neu antibody depends on both innate and adaptive immunity. Cancer Cell 2010;18:160-70.

3. Clynes R, Knutson KL, Ballman KV, Erskine CL, Norton N, Sumrall SV, et al. Combination trastuzumab and chemotherapy to induce immunity to multiple tumor antigens in patients with HER2-positive metastatic breast cancer: NCCTG (Alliance) studies N0337 and N98-32-52. ASCO Meeting Abstracts 2013;31:521.

4. Taylor C, Hershman D, Shah N, Suciu-Foca N, Petrylak DP, Taub R, et al. Augmented HER-2 specific immunity during treatment with trastuzumab and chemotherapy. Clin Cancer Res 2007;13:5133-43.

5. Demaria S, Pilones KA, Formenti SC, Dustin ML. Exploiting the stress response to radiation to sensitize poorly immunogenic tumors to antiCTLA-4 treatment. Oncoimmunology 2013;2:e23127.

6. Salgado R, Denkert C, Demaria S, Sirtaine N, Klauschen F, Pruneri G, et al. The evaluation of tumor-infiltrating lymphocytes (TILs) in breast cancer: recommendations by an International TILs Working Group 2014. Ann Oncol 2015;26:259-71.

7. Herbst RS, Soria JC, Kowanetz M, Fine GD, Hamid O, Gordon MS, et al Predictive correlates of response to the anti-PD-L1 antibody MPDL3280A in cancer patients. Nature 2014;515:563-7.

8. Gajewski TF. Cancer immunotherapy. Mol Oncol 2012;6:242-50.

9. Denkert C, Loibl S, Noske A, Roller M, Muller BM, Komor M, et al. Tumor-associated lymphocytes as an independent predictor of response to neoadjuvant chemotherapy in breast cancer. J Clin Oncol 2010;28: 105-13.

10. Loi S, Michiels S, Lambrechts D, Fumagalli D, Claes B, KellokumpuLehtinen PL, et al. Somatic mutation profiling and associations with prognosis and trastuzumab benefit in early breast cancer. J Natl Cancer Inst 2013;105:960-7.

\section{Authors' Contributions}

Conception and design: D.B. Page, J. Yuan, J.C. Durack, S. Solomon, Z. Dong, A. Diab, J. Sung, M. Maybody, V. Sacchini, S. Patil, J.P. Allison, J.D. Wolchok, C. Hudis, L. Norton, H. McArthur

Development of methodology: D.B. Page, J. Yuan, J.C. Durack, R. Emerson, S. Solomon, Z. Dong, V. Sacchini, H. Robins, H. McArthur

Acquisition of data (provided animals, acquired and managed patients, provided facilities, etc.): D.B. Page, J. Yuan, J.C. Durack, R. Emerson, S. Solomon, P. Wong, C. Comstock, A. Diab, J. Sung, M. Maybody, E. Brogi, V. Sacchini, H. Robins, C. Hudis, H. McArthur

Analysis and interpretation of data (e.g., statistical analysis, biostatistics, computational analysis): D.B. Page, J. Yuan, D. Redmond, Y.H. Wen, J.C Durack, R. Emerson, S. Solomon, A. Diab, V. Sacchini, O. Elemento, H. Robins, S. Patil, J.D. Wolchok, H. McArthur

Writing, review, and/or revision of the manuscript: D.B. Page, J. Yuan, D. Redmond, Y.H. Wen, J.C. Durack, S. Solomon, P. Wong, C. Comstock, A. Diab, J. Sung, M. Maybody, E. Morris, E. Brogi, M. Morrow, V. Sacchini, S. Patil, J.D. Wolchok, C. Hudis, H. McArthur

Administrative, technical, or material support (i.e., reporting or organizing data, constructing databases): D.B. Page, S. Solomon, E. Brogi, V. Sacchini, C. Hudis, H. McArthur

Study supervision: J. Yuan, S. Solomon, V. Sacchini, C. Hudis, H. McArthur

\section{Grant Support}

Salary support of the primary author (Dr. David Page) was funded by the Terri Brodeur Breast Cancer Foundation Fellowship Grant. Costs of completing the ImmunoSEQ assay were sponsored by Adaptive Biotechnologies.

The costs of publication of this article were defrayed in part by the payment of page charges. This article must therefore be hereby marked advertisement in accordance with 18 U.S.C. Section 1734 solely to indicate this fact.

Received February 2, 2016; revised July 19, 2016; accepted August 3, 2016; published OnlineFirst September 1, 2016.

11. Waitz R, Solomon SB, Petre EN, Trumble AE, Fasso M, Norton L, et al. Potent induction of tumor immunity by combining tumor cryoablation with anti-CTLA-4 therapy. Cancer Res 2012;72:430-9.

12. Leach DR, Krummel MF, Allison JP. Enhancement of antitumor immunity by CTLA-4 blockade. Science 1996;271:1734-6.

13. Gage AA, Baust JM, Baust JG. Experimental cryosurgery investigations in vivo. Cryobiology 2009;59:229-43

14. Robert C, Thomas L, Bondarenko I, O'Day S, M DJ, Garbe C, et al. Ipilimumab plus dacarbazine for previously untreated metastatic melanoma. N Engl J Med 2011;364:2517-26.

15. Hodi FS, O'Day SJ, McDermott DF, Weber RW, Sosman JA, Haanen JB, et al. Improved survival with ipilimumab in patients with metastatic melanoma N Engl J Med 2010;363:711-23.

16. Egen JG, Kuhns MS, Allison JP. CTLA-4: new insights into its biological function and use in tumor immunotherapy. Nat Immunol 2002;3:611-8

17. Simpson TR, Li F, Montalvo-Ortiz W, Sepulveda MA, Bergerhoff K, Arce F, et al. Fc-dependent depletion of tumor-infiltrating regulatory $\mathrm{T}$ cells codefines the efficacy of anti-CTLA-4 therapy against melanoma. J Exp Med 2013;210:1695-710.

18. Tumeh PC, Harview CL, Yearley JH, Shintaku IP, Taylor EJ, Robert L, et al PD-1 blockade induces responses by inhibiting adaptive immune resistance. Nature 2014;515:568-71.

19. Diab A, McArthur HL, Solomon SB, Sacchini V, Comstock C, Maybody M, et al. A pilot study of preoperative (Pre-op), single-dose ipilimumab (Ipi) and/or cryoablation (Cryo) in women (pts) with early-stage/resectable breast cancer (ESBC). ASCO Meeting Abstracts 2014;32:1098

20. Robins HS, Campregher PV, Srivastava SK, Wacher A, Turtle CJ, Kahsai O, et al. Comprehensive assessment of T-cell receptor beta-chain diversity in alphabeta T cells. Blood 2009;114:4099-107.

21. Adams S, Gray RJ, Demaria S, Goldstein L, Perez EA, Shulman LN, et al. Prognostic value of tumor-infiltrating lymphocytes in triple-negative breast cancers from two phase III randomized adjuvant breast cancer trials: ECOC 2197 and ECOG 1199. J Clin Oncol 2014;32:2959-66. 
22. Sherwood AM, Emerson RO, Scherer D, Habermann N, Buck K, Staffa J, et al. Tumor-infiltrating lymphocytes in colorectal tumors display a diversity of $\mathrm{T}$ cell receptor sequences that differ from the $\mathrm{T}$ cells in adjacent mucosal tissue. Cancer Immunol Immunother 2013;62:1453-61.

23. Cha E, Klinger M, Hou $Y$, Cummings $C$, Ribas A, Faham M, et al. Improved survival with T cell clonotype stability after anti-CTLA-4 treatment in cancer patients. Sci Transl Med 2014;6:238ra70.

24. Loi S, Michiels S, Salgado R, Sirtaine N, Jose V, Fumagalli D, et al. Tumor infiltrating lymphocytes are prognostic in triple negative breast cancer and predictive for trastuzumab benefit in early breast cancer: results from the FinHER trial. Ann Oncol 2014;25:1544-50.

25. Sabel MS. Cryo-immunology: a review of the literature and proposed mechanisms for stimulatory versus suppressive immune responses. Cryobiology 2009;58:1-11.

26. Emens LA. Inhibition of PD-L1 by MPDL3280A leads to clinical activity in patients with metastatic triple-negative breast cancer
(TNBC). AACR Annual Meeting; April 20, 2015; Philidelphia, PA; 2015.

27. Nanda R, Chow LQ, Dees EC, Berger R, Gupta S, Geva R, et al. A phase Ib study of pembrolizumab (MK-3475) in patients with advanced triplenegative breast cancer. San Antonio Breast Cancer Symposium; December 8-13, 2014; San Antonio, TX; 2014.

28. Dirix LY, Takacs I, Nikolinakos P, Jerusalem G, Arkenau H-T, Hamilton EP, et al. Avelumab (MSB00107118C), an anti-PD-L1 antibody, in patient with locally advanced or metastatic breast canceR: A phase Ib JAVELIN solid tumor trial. San Antonio Breast Cancer Symposium; December 8-12, 2015; San Antonio, TX; 2015.

29. Rugo H, DeLord JP, Im S-A, Ott PA, Piha-Paul SA, Bedard PL, et al Preliminary efficacy and safety of pembrolizumab (MK-3475) in patients with PD-L1-positive, estrogen receptor positive (ER+)/HER2-negative advanced breast cancer enrolled in KEYNOTE-028. San Antonio Breast Cancer Symposium; December 8-12, 2015; San Antonio, TX; 2015. 
Correction: Deep Sequencing of T-cell

\section{Receptor DNA as a Biomarker of Clonally} Expanded TILs in Breast Cancer after Immunotherapy

In the article by Page and colleagues (Cancer Immunol Res 2016; 4:835-44), which appeared in the October 2016 issue of Cancer Immunology Research (1), the table of TCR sequences from breast cancer samples was inadvertently omitted. The table is now included in the article as Supplementary Table S1. Data analyses were performed using the ImmunoSEQ Analyzer provided by Adaptive Technologies and the data are available at https://clients.adaptivebiotech.com/pub/page-2016-cir. The authors regret these omissions.

\section{Reference}

1. Page DB, Yuan J, Redmond D, Wen YH, Durack JC, Emerson R, et al. Deep sequencing of T-cell receptor DNA as a biomarker of clonally expanded TILs in breast cancer after immunotherapy. Cancer Immunol Res 2016;4:835-44.

Published online March 1, 2017.

doi: 10.1158/2326-6066.CIR-17-0048

(2)17 American Association for Cancer Research. 


\section{Cancer Immunology Research}

\section{Deep Sequencing of T-cell Receptor DNA as a Biomarker of Clonally Expanded TILs in Breast Cancer after Immunotherapy}

David B. Page, Jianda Yuan, David Redmond, et al.

Cancer Immunol Res 2016;4:835-844. Published OnlineFirst September 1, 2016.

Updated version Access the most recent version of this article at:

doi:10.1158/2326-6066.CIR-16-0013

Supplementary Access the most recent supplemental material at:

Material http://cancerimmunolres.aacrjournals.org/content/suppl/2017/01/28/2326-6066.CIR-16-0013.DC1

Cited articles This article cites 25 articles, 8 of which you can access for free at:

http://cancerimmunolres. aacrjournals.org/content/4/10/835.full\#ref-list-1

Citing articles This article has been cited by 9 HighWire-hosted articles. Access the articles at:

http://cancerimmunolres. aacrjournals.org/content/4/10/835.full\#related-urls

E-mail alerts Sign up to receive free email-alerts related to this article or journal.

Reprints and To order reprints of this article or to subscribe to the journal, contact the AACR Publications Department Subscriptions at pubs@aacr.org.

Permissions To request permission to re-use all or part of this article, use this link http://cancerimmunolres.aacrjournals.org/content/4/10/835.

Click on "Request Permissions" which will take you to the Copyright Clearance Center's (CCC)

Rightslink site. 